\title{
Neue «Bench-to-Bed»-Ansätze in der Verhaltensmedizin
}

Der enorme Erkenntniszuwachs in den Neurowissenschaften ist für die Diagnostik und Behandlung von psychischen und psychosomatischen Behandlungen von größter Bedeutung. So zeichnen sich für die Behandlung von neurologischen und psychiatrischen Krankheiten wirklich innovative und erfolgversprechende Ansätze ab. Wie steht es aber mit den Bereichen Verhaltenstherapie und Verhaltensmedizin?

Ein Blick in Zeitschriften wie Psychosomatic Medicine, Health Psychology, Journal of Psycho-Somatic Research oder Psychotherapy and Psychosomatics zeigt, dass in aller Regel korrelative Studien durchgeführt werden. Derartige Untersuchungen zeigen, dass klinisch relevante psychologische Parameter parallel zu endokrinologischen, immunologischen und autonomen Maßen variieren. Darüber ergeben sich Hinweise auf Subgruppen, die sich hinsichtlich dieser Parameter unterscheiden und womöglich auch anderer Interventionsmöglichkeiten bedürfen. Es liegen auch einige wenige Untersuchungen vor, welche zeigen, dass psychotherapeutische Interventionen positiv auf derartige physiologische Systeme einwirken können. Daraus lassen sich erste Überlegungen zu mechanistischen Zusammenhängen ableiten. Eine besondere Bedeutung kommt hier bildgebenden Verfahren zu, mit denen geprüft werden kann, ob sich störungsspezifische zentralnervöse Veränderungen durch verhaltenstherapeutische Interventionen verändern lassen.

Bei psychosomatischen Störungen ist die Kluft zwischen neurobiologischer Grundlagenforschung und verhaltensmedizinischer Praxis besonders groß. Die Komplexität der Mechanismen umfasst hier nicht nur endokrinologische, immunologische, autonome und zentralnervöse Abläufe, sondern auch pathologische Vorgänge in Organsystemen. Aus dieser Situation ergibt sich zwangsläufig, dass derartige Fragestellungen nur im interdisziplinären Verbund kompetent bearbeitet werden können. Allerdings ist es kaum möglich, den wissenschaftlichen und therapeutischen Nachwuchs adäquat auszubilden.
Zur Jahreswende hat die Universität Trier erstmals ein Postgraduierten-Studiengang eingerichtet. Sechs neue Professuren wurden geschaffen, um Doktorandinnen und Doktoranden der Psychologie, Medizin, Biologie usw. neben der Dissertation eine angemessene Ausbildung in einer neurobiologisch orientierten Verhaltensmedizin zu ermöglichen. In den USA und Kanada wurden in den letzten Jahren vielerorts vergleichbare «Brain-body»-Zentren eingerichtet. In diesen Einrichtungen, die sich zumeist auf bestimmte Fragestellungen spezialisiert haben, wird versucht, in enger fachübergreifender $\mathrm{Zu}$ sammenarbeit Methoden und Kenntnisse der Hirnforschung zu nutzen, um körperliche Erkrankungen besser verstehen und behandeln zu können.

Auch wenn sich damit die Situation für den klinischen und wissenschaftlichen Nachwuchs verbessert, so ändert dies zunächst nichts an dem bemerkenswerten Missstand, dass neurobiologisches Wissen bisher kaum für die klinische Praxis der Verhaltensmedizin genutzt wird. Der nahezu unübersehbaren Fülle an neurobiologischen Informationen zu krankheitsrelevanten Mechanismen, die bisher in der Literatur veröffentlicht wurden, steht die profunde Hilflosigkeit gegenüber, dieses Wissen klinisch nutzbar zu machen. Die International Society for Psychoneuroendocrinology (ISPNE) hat daher in diesem Jahr begonnen, eine umfassende Initiative zu starten, um endlich wirksame klinische Fortschritte zu erzielen. Die ISPNE gründet derzeit verschiedene Arbeitsgruppen mit führenden Fachwissenschaftlern und Fachwissenschaftlerinnen aus Klinik und Grundlagenforschung. Diese Arbeitsgruppen haben die Aufgabe zu prüfen, welche Erkenntnisse und Messverfahren aus bestimmten Bereichen der Grundlagenforschung geeignet sind, um die Diagnostik und Therapie von psychosomatischen Erkrankungen zu verbessern. Auf den Jahreskongressen dieser Fachgesellschaft sollen die Arbeitsgruppen ihre Ergebnisse vortragen. Dies wird erstmals im September 2003 auf dem Jahreskongress in New York gesche-

\section{KARGER (c) 2002 S. Karger GmbH, Freiburg


hen. Die ISPNE beabsichtigt darüber hinaus, ab 2004 regelmäßig entsprechende Empfehlungen zu veröffentlichen. $\mathrm{Ob}$ diese Initiative erfolgreich ist, wird sich erst in den nächsten Jahren zeigen. Mit etwas Optimismus kann man aber davon ausgehen, dass die Verhaltensmedizin sehr wirksam, schrittweise und systematisch verbessert wird. Es gibt wohl auch keine Alternative zu diesem Ansatz, wenn man nicht noch länger warten will, das Wissen der Hirnforschung für die Verhaltensmedizin nutzbar zu machen.
Unsere Zeitschrift wird diese Entwicklung verfolgen und regelmäßig über die Fortschritte informieren. Es bleibt zu wünschen, dass diese Entwicklungen auch in der universitären Ausbildung der Studierenden und in der verhaltenstherapeutischen Weiterbildung angemessen berücksichtigt werden. Denn nur so kann letztendlich eine erfolgreiche Umsetzung derartiger Konzepte in die Praxis erfolgen.

D. Hellhammer, Trier 